\title{
Surgical Excision Followed by Pulsed Dye Laser Sessions for the Successful Treatment of Glomangiomatosis on the Left Flank: A Case Report and Literature Review
}

\section{Sooyeon Park, Sang-oon Baek, En Young Rha, Jun Yong Lee, Hyun Ho Han}

Department of Plastic and Reconstructive Surgery, Incheon St. Mary's Hosipital, College of Medicine, The Catholic University of Korea, Incheon, Korea

No potential conflict of interest relevant to this article was reported.
Glomus tumors are benign neoplasms that are usually found in the subungual area, and are painful upon palpation. The typical treatment of choice is surgical excision. In rare cases, glomus tumors are observed as large-sized clusters, and are classified as atypical tumors known as glomangiomatosis. While surgical excision is the accepted standard of care, this proves difficult because of the tumors' large size and satellite lesions. We present the successful treatment of asymptomatic glomangiomatosis is situated on the left flank area by a combination of surgical excision and pulsed dye laser, which minimized the risk of scar formation.

Keywords Glomus tumor, Lasers dye, Neoplasm, Scar

\section{INTRODUCTION}

Glomus tumors are perivascular neoplasms that originate from perivascular muscle cells known as glomic cells [1]. Occasionally, glomus tumors display unusual features, such as manifesting in the form of multiple large clusters throughout the body. Additionally, they can be composed of well-developed large and small vessels, and such tumors form an atypical subtype known as glomangiomatosis [2].

In this study, we present a case of a biopsy-proven glomangiomatosis on the left flank that was successfully treated by a combination of surgical excision of the dominant lesion and subsequent pulsed dye laser (PDL) sessions on the remnant satellite lesions.

Received: May 15, 2016 Revised: Jun 15, 2016 Accepted: Jun 16, 2016 Correspondence: Hyun Ho Han Department of Plastic and Reconstructive Surgery, Seoul St. Mary's Hospital, College of Medicine, The Catholic University of Korea, 222 Banpo-daero, Seocho-gu, Seoul 06591, Korea. E-mail: tripleH@catholic.ac.kr

Copyright () 2016 The Korean Society for Aesthetic Plastic Surgery.

This is an Open Access article distributed under the terms of the Creative Commons Attribution Non-Commercial License (http://creativecommons.org/licenses/by-nc/4.0/) which permits unrestricted non-commercial use, distribution, and reproduction in any medium, provided the original work is properly cited. $\quad w w w . e-a a p s . o r g$

\section{CASE}

A 46-year-old woman presented with a $5.0 \times 2.0-\mathrm{cm}$ mass in the left flank area (Fig. 1A). Since the mass was asymptomatic, the patient had not received any treatment over the course of the previous 30 years. A contrast-enhanced computed tomography (CT) scan revealed a $4.5 \times 1.7-\mathrm{cm}$ multilobulated mass with peripheral nodular enhancement, accompanied by satellite lesions in the skin and the subcutaneous layer. The mass was suspected to be a hemangioma (Fig. 1B).

Surgery was performed under local anesthesia, with the incision line made on the dominant lesion. To minimize scar formation, the satellite nodules were not removed from the subcutaneous layer together with the dominant lesion.

A biopsy was performed, and the results of a histopathological analysis using hematoxylin and eosin staining showed that the mass was composed of round epithelioid cells proliferating around large and small capillaries (Fig. 2A and 2B). Since those pathologic findings suggested that the excised mass was a glomus tumor instead of a hemangioma, as suspected prior to surgery, immunohistochemical staining was performed in the pathology department. The tumor cells stained positive for smooth-muscle actin and CD-34. These results, in combination with the pathologic findings of angiomatosis, demonstrated that this was a case of glomangiomatosis (Fig. 2C 

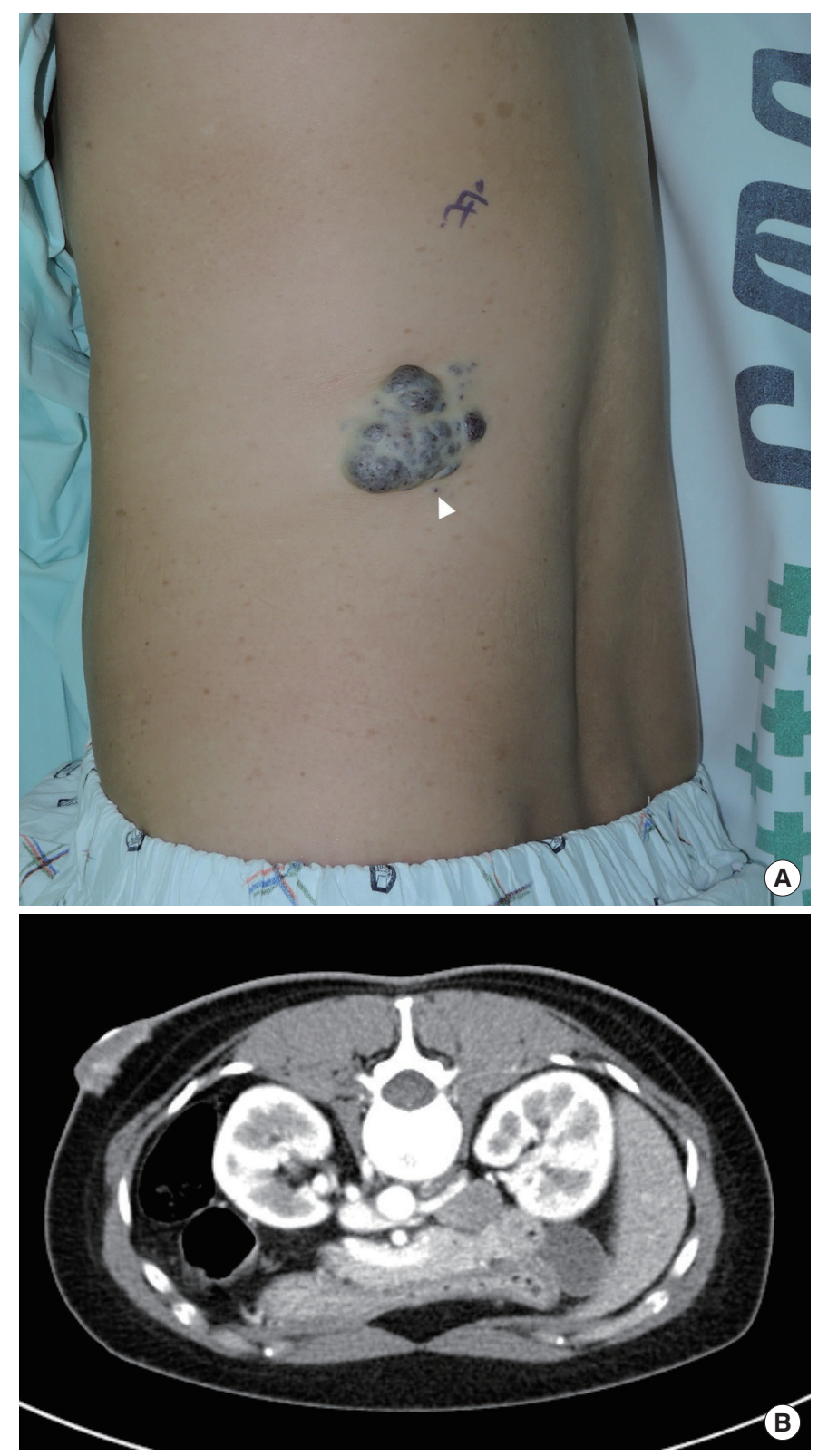

Fig. 1. (A) A preoperative clinical photograph shows multiple purplish nodular lesions on the left flank area with satellite lesions (arrow). (B) A preoperative computed tomography image of the left flank shows multilobulated lesions with peripheral nodular enhancement.

and 2D). Starting 1 month after the surgery, PDL sessions were administered twice per week for 2 months in the outpatient department to treat the remnant satellite lesions (Fig. 3A). We used a $7-\mathrm{mm}$ spot size, $0.45 \mathrm{~ms}$ pulse duration $\left(6-8 \mathrm{~J} / \mathrm{cm}^{2}\right)$, and a wavelength of $595 \mathrm{~nm}$. Although purpura was observed, it subsided within a week. Following the surgical removal of the main tumor and the subsequent PDL therapy, an 18-month postoperative follow-up examination found no recurrent lesions in the treated region (Fig. 3B).

\section{DISCUSSION}

Glomus tumors are rare benign tumors that originate from glomic cells [1]. Glomic cells are located in arteriovenous anastomoses and are responsible for thermoregulation [2]. The typical size of such tumors is between $5 \mathrm{~mm}$ and $1 \mathrm{~cm}$, and they are characterized by reddish-purple discoloration in the subungal or subcutaneous areas [3].

Glomus tumors have 3 major components: glomic cells, blood vessels, and smooth muscle. They are divided into 3 types depending on the predominant type of cell that forms the tumor: glomangioma, if vascular cells are predominant; solid glomus tumor, if glomic cells are predominant; and glomangiomyoma, if smooth muscle cells are predominant [1]. It is difficult to distinguish between a glomus tumor in general and a solid glomus tumor. In general glomus tumors are also known as glomuvenous malformations. Glomangiomatosis refers to a glomus tumor that contains large and small vessels with the pathologic findings of angiomatosis. Therefore, glomangiomatosis is an atypical subtype of glomuvenous malformations [2].

Glomangiomatosis is rare, accounting for $2 \%$ to $3 \%$ of glomus tumors. Such tumors are usually large and are not observed in the subungal area [1,2]. No instances of metastasis or malignant changes have been reported in these tumors, and they mainly occur in the upper and lower extremities, and rarely on the head and neck, chest wall, or the paravertebral area [4-6].

Histopathological analysis is essential for a precise diagnosis. The typical histological features of glomus tumors include an internal endothelial lining and non-uniform vessels surrounded by glomic cells that are situated in the deep dermal layer [5]. In the case of pain-inducing tumors, a nonmedullated nerve fiber is observed near the glomus cells [7]. In glomangiomatosis, large and small vessels show infiltrative proliferation, referred to as angiomatosis, and are surrounded by the glomus cells [5]. In immunohistochemical analyses, the tumor cells stain positive for actin (up to 99\%) and CD-34 (up to $32 \%$ ) $[5,8]$.

The most commonly performed treatment of glomangiomatosis is resection. For a large mass, this means that the main lesion is excised together with any satellite lesions that may be present. Such surgical procedures have esthetic consequences. To avoid making the excision line wider, leading to subsequent scar formation, we propose combining the surgical excision of the main lesion with laser treatment of the small adjacent lesions. This proved highly effective in our case.

Multiple trials of laser radiation therapy (e.g., argon, carbon dioxide, neodymium-doped yttrium aluminium garnet) on small superficial glomus tumors have been previously documented in the literature [9]. PDL targets the vessels and causes minimal damage to the dermal and epidermal structures. It is currently used for treating hemangiomas and port-wine nevus in children $[10,11]$. In 

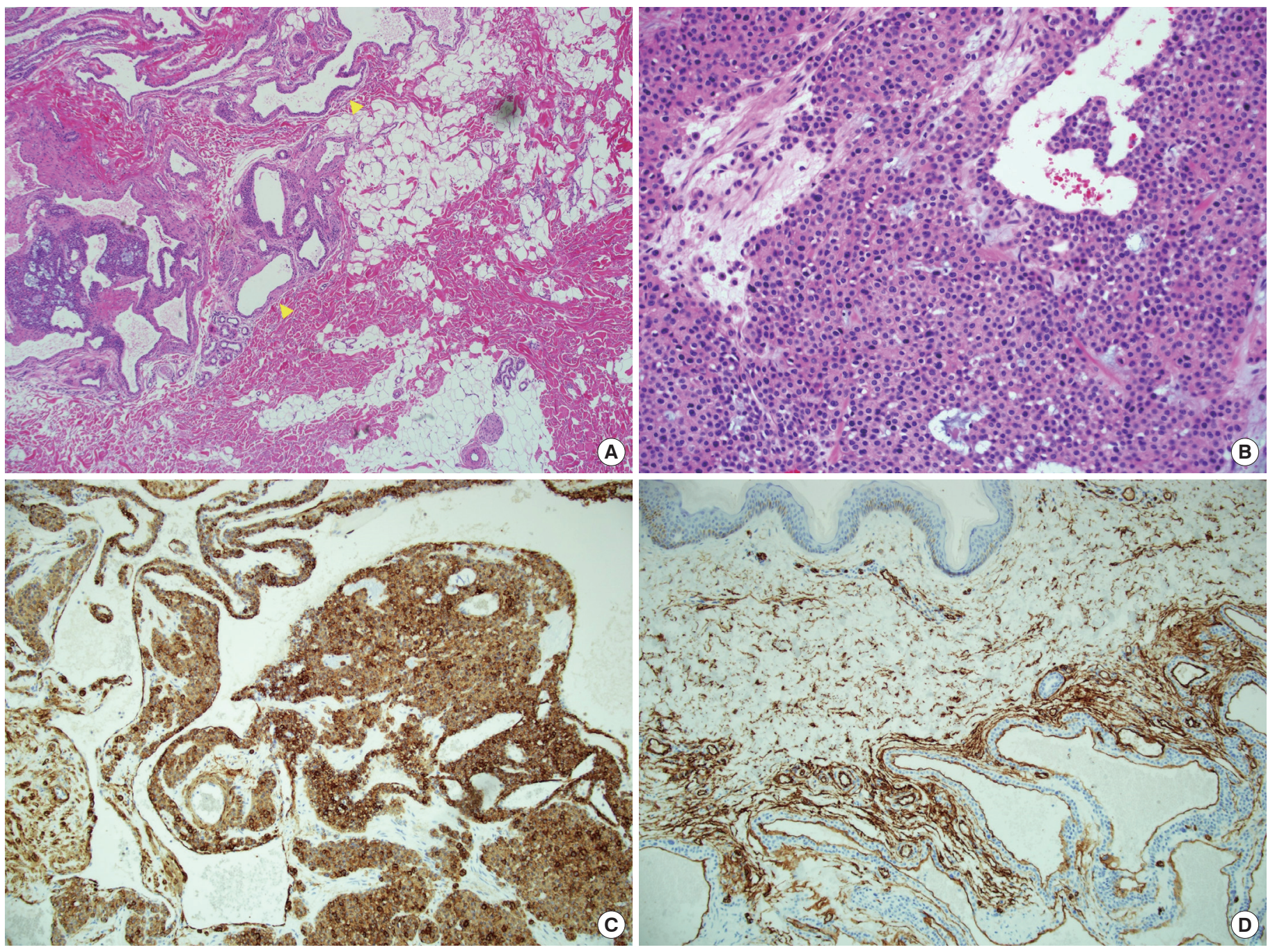

Fig. 2. Histopathological findings. (A) A tumor composed of scattered and dilated vascular spaces (yellow arrow) (hematoxylin and eosin stain $[H \& E], \times 40)$. (B) Largely dilated vascular spaces and perivascular infiltration of glomic cells (H\&E, $\times 200$ ). (C) Positive staining for the expression of smooth-muscle actin antibody. (D) Positive staining for the expression of CD-34 in the vascular space.

addition, a small subungal glomus tumor was successfully treated using PDL [12].

In the case presented here, which involved glomangiomatosis with multiple large adjacent lesions, the two-step treatment resulted in excellent esthetic outcomes. First, the main mass was surgically excised. Second, to minimize the surgical excision margin, the PDL technique was performed in the outpatient department. Our results confirmed that PDL therapy effectively removed the dilated vessels in the satellite lesion while causing little damage to the surrounding tissue. Furthermore, it may increase the likelihood of recurrent glomus tumors. The cellular mechanism of PDL therapy has yet to be fully elucidated. While further studies are required, it is most likely that cellular apoptosis, microvascular destruction, and inflammation are responsible for the tissue reaction following PDL [10].
In summary, surgical excision of the dominant lesion combined with PDL treatment of the satellite lesions was found to be an effective method for treating a case of glomangiomatosis involving multiple large lesions, while minimizing postoperative scar formation.

\section{PATIENT CONSENT}

Patients provided written consent for the use of their images.

\section{REFERENCES}

1. Cabral CR, Oliveira Filho J, Matsumoto JL, et al. Type 2 segmental glomangioma-Case report. An Bras Dermatol 2015;90:97-100.

2. Hiruta N, Kameda N, Tokudome T, et al. Malignant glomus tumor: a case report and review of the literature. Am J Surg Pathol 1997;21:1096- 

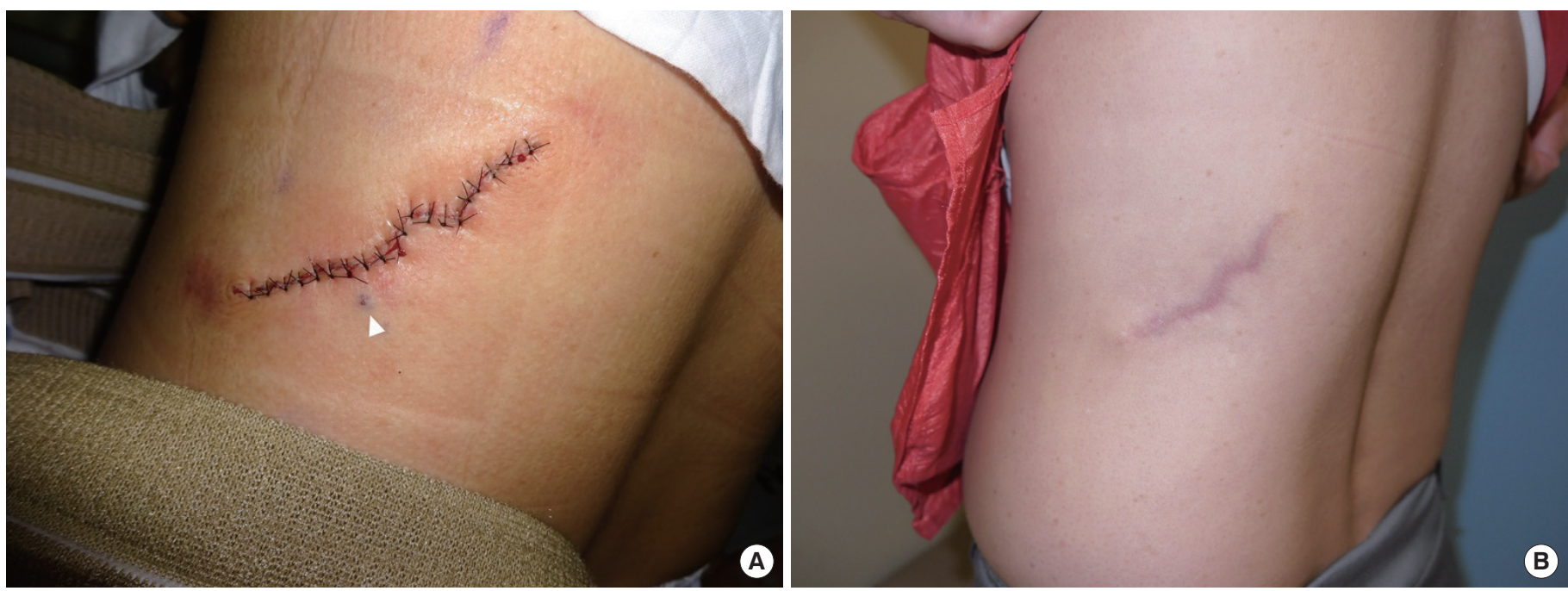

Fig. 3. Application of pulsed dye laser treatment on the left flank area. (A) Immediate postoperative clinical photograph demonstrating remnant satellite nodular lesions below the incision (arrow). (B) Clinical photo after laser treatment, with no remnant lesions.

103.

3. Lee DY, Hwang SC, Jeong ST, et al. The value of diagnostic ultrasonography in the assessment of a glomus tumor of the subcutaneous layer of the forearm mimicking a hemangioma: a case report. J Med Case Rep 2015;9:191.

4. Park EA, Hong SH, Choi JY, et al. Glomangiomatosis: magnetic resonance imaging findings in three cases. Skeletal Radiol 2005;34:108-11.

5. Folpe AL, Fanburg-Smith JC, Miettinen M, et al. Atypical and malignant glomus tumors: analysis of 52 cases, with a proposal for the reclassification of glomus tumors. Am J Surg Pathol 2001;25:1-12.

6. Sano K, Hosaka K, Ozeki S. Glomangiomatosis concentrated in the ankle with varied appearances: a case report. J Foot Ankle Surg 2014; 53:468-71.

7. Heys SD, Brittenden J, Atkinson P, et al. Glomus tumour: an analysis of 43 patients and review of the literature. Br J Surg 1992;79:345-7.
8. Mravic M, LaChaud G, Nguyen A, et al. Clinical and histopathological diagnosis of glomus tumor: an institutional experience of 138 cases. Int J Surg Pathol 2015;23:181-8.

9. Barnes L, Estes SA. Laser treatment of hereditary multiple glomus tumors. J Dermatol Surg Oncol 1986;12:912-5.

10. Cao Y, Wang F, Jia Q, et al. One possible mechanism of pulsed dye laser treatment on infantile hemangioma: induction of endothelial apoptosis and serum vascular endothelial growth factor (VEGF) level changes. J Lasers Med Sci 2014;5:75-81.

11. Chang HS, Kim YG, Lee JH. Treatment using a long pulsed nd:yag laser with a pulsed dye laser for four cases of blebbed port wine stains. Ann Dermatol 2011;23 Suppl 1:S75-8.

12. Vergilis-Kalner IJ, Friedman PM, Goldberg LH. Long-pulse 595-nm pulsed dye laser for the treatment of a glomus tumor. Dermatol Surg 2010;36:1463-5. 